\title{
Some invariant solutions and conservation laws of a type of long-water wave system
}

Xiangzhi Zhang ${ }^{1 *}$ (1) and Yufeng Zhang ${ }^{1}$

"Correspondence: zz301@163.com

'School of Mathematics, China University of Mining and

Technology, Xuzhou, P.R. China

\begin{abstract}
We propose a generalized long-water wave system that reduces to the standard water wave system. We also obtain the Lax pair and symmetries of the generalized shallow-water wave system and single out some their similarity reductions, group-invariant solutions, and series solutions. We further investigate the corresponding self-adjointness and the conservation laws of the generalized system.
\end{abstract}

PACS Codes: 05.45.Yv; 02.30.Jr; 02.30.Ik

Keywords: Similarity solution; Conservation law; Symmetry

\section{Introduction}

The classical dispersiveless long wave equations

$$
\left\{\begin{array}{l}
u_{t}+u u_{x}+h_{x}=0 \\
h_{t}+(u h)_{x}=0
\end{array}\right.
$$

have a number of dispersive generalizations [1]. Kupershmidt [2] considered the following extension of (1):

$$
\left\{\begin{array}{l}
u_{t}=\left(\frac{1}{2} u^{2}+h-\beta u_{x}\right)_{x}, \\
h_{t}=\left(u h+\alpha u_{x x}-\beta h_{x}\right)_{x},
\end{array}\right.
$$

where $\alpha, \beta$ are arbitrary constants. The invertible change of variables $u=\bar{u}, h=\bar{h}+\gamma \bar{u} x$, turns (2) into

$$
\left\{\begin{array}{l}
\bar{u}_{t}=\left(\frac{1}{2} \bar{u}^{2}+\bar{h}+\mu \bar{u}_{x}\right)_{x}, \\
\bar{h}_{t}=\left(\bar{u} \bar{h}-\mu \bar{h}_{x}\right)_{x}, \mu=\gamma+\beta= \pm \sqrt{\alpha+\beta^{2}} .
\end{array}\right.
$$

Broer [1] derived system (2) for $\alpha=\frac{1}{3}, \beta=0$, for which it is the proper Boussinesq equation. In terms of the potential $\varphi: u=\varphi_{x}$, system (2) was derived by Kaup [3]. Later, Matveev and Yavor [4] found algebrogeometrically a large class of almost periodic solutions. Li, Ma,

(c) The Author(s) 2019. This article is distributed under the terms of the Creative Commons Attribution 4.0 International License (http://creativecommons.org/licenses/by/4.0/), which permits unrestricted use, distribution, and reproduction in any medium, provided you give appropriate credit to the original author(s) and the source, provide a link to the Creative Commons license, and indicate if changes were made. 
and Zhang [5] used a scaling transformation to transfer a nonlinear long wave equation of Boussinesq class to the Broer-Kaup (BK) system, a type of long water wave equations:

$$
\left\{\begin{array}{l}
v_{t}=\frac{1}{2}\left(v^{2}+2 w-v_{x}\right)_{x}, \\
w_{t}=\left(v w+\frac{1}{2} w_{x}\right)_{x} .
\end{array}\right.
$$

Furthermore, some exact solutions and Darboux transformations of (1) were obtained by applying the Lax-pair method. In terms of [5], we can study the similarity reduction, exact solutions, and conservation laws of the Boussinesq system through the scalar transformation

$$
v=-u, w=\xi+1+\frac{v_{x}}{2}
$$

that is, we can transform the BK system to the Boussinesq system

$$
\left\{\begin{array}{l}
\xi_{t}+[(1+\xi) u]_{x}=-\frac{1}{4} u_{x x x} \\
u_{t}+u u_{x}+\xi_{x}=0
\end{array}\right.
$$

where $\xi$ is the elevation of the water wave, $u$ is the surface velocity of water along the $x$-direction. Hence, the results of the paper have certain physical sense.

In the paper, we construct a generalized BK system as follows:

$$
\left\{\begin{array}{l}
v_{t}=\frac{\alpha}{2}\left(v_{x}-v^{2}-2 w\right)_{x}-\beta v_{x} \\
w_{t}=-\frac{\alpha}{2}\left(w_{x}+2 w v\right)_{x}-\beta w_{x}
\end{array}\right.
$$

where $\alpha, \beta$ are constants, so that some symmetries of (2) are produced by the symmetry group method [6]. It follows that some similarity solutions, group-invariant solutions, and series solutions are produced. In addition, Ibragimov and Avdonina [7] showed how to apply the symmetries of differential equations to study the self-adjointness and conservation laws. Thus we would like to follow the approach to investigate the quasiself-adjointness and conservation laws of the generalized BK system (2).

\section{Integrability of (2)}

Set

$$
\varphi_{x}=U \varphi, \quad \varphi_{t}=V \varphi,
$$

where

$$
\begin{aligned}
& U=\left(\begin{array}{cc}
-\lambda+\frac{\nu}{2} & 1 \\
-w & \lambda-\frac{\nu}{2}
\end{array}\right), \\
& V=\left(\begin{array}{cc}
\alpha \lambda^{2}+\beta \lambda+\frac{\alpha}{4} v_{x}-\frac{\alpha}{4} \nu^{2}-\frac{\beta}{2} \nu & -\alpha \lambda-\frac{\alpha}{2} \nu-\beta \\
\alpha w \lambda+\frac{\alpha}{2} w_{x}+\frac{\alpha}{2} w \nu+\beta w & -\alpha \lambda^{2}-\beta \lambda-\frac{\alpha}{4} v_{x}+\frac{\alpha}{4} v^{2}+\frac{\beta}{2} \nu
\end{array}\right) .
\end{aligned}
$$


Then the compatibility condition of (3)

$$
V_{t}-U_{x}+U V-V U=0
$$

admits the generalized BK system, which can be directly verified. Hence the generalized BK system (2) is Lax integrable. Using (3), we can get some Darboux transformations for deducing solutions of the system. Here we omit them.

\section{Similarity solutions and group-invariant solutions}

Applying the Lie symmetry analysis, we can get the symmetry of system (2):

$$
X_{1}=\frac{\partial}{\partial t}, \quad X_{2}=\frac{\partial}{\partial x}, \quad X_{3}=\left(\frac{1}{2} x+\beta t\right) \frac{\partial}{\partial x}+t \frac{\partial}{\partial t}-\frac{1}{2} v \frac{\partial}{\partial v}-w \frac{\partial}{\partial w} .
$$

The vector field $X_{3}$ has the following characteristic equation:

$$
\frac{d t}{t}=\frac{d x}{\frac{1}{2} x+\beta t}=\frac{d v}{-\frac{1}{2} v}=\frac{d w}{-w}
$$

which gives rise to

$$
\left(\beta t+\frac{1}{2} x\right) d t-t d x=0
$$

One integration factor of (6) is given by

$$
\mu=e^{-\int \frac{3}{2 t} d t}=t^{-\frac{3}{2}},
$$

which transfers (6) to the complete integration equation

$$
\beta t^{-\frac{1}{2}} d t+d\left(-t^{-\frac{1}{2}} x\right)=0
$$

from which we have the invariant variable $\xi=2 \beta t^{\frac{1}{2}}-t^{-\frac{1}{2}} x$. In terms of Eq. (5), we have the formal invariants

$$
v=t^{-\frac{1}{2}} f(\xi), \quad w=t^{-1} g(\xi)
$$

where $f(\xi)$ and $g(\xi)$ are arbitrary smooth functions of $\xi$. Substituting (7) into system (2) yields the ordinary differential system

$$
\left\{\begin{array}{l}
-\frac{1}{2} f(\xi)+\frac{1}{2} x t^{-\frac{1}{2}} f^{\prime}(\xi)=\frac{\alpha}{2}\left[f^{\prime \prime}(\xi)+2 f(\xi) f^{\prime}(\xi)+2 g^{\prime}(\xi)\right] \\
-g(\xi)+\frac{1}{2} x t^{-\frac{1}{2}} g^{\prime}(\xi)=-\frac{\alpha}{2}\left[g^{\prime \prime}(\xi)-2 g^{\prime}(\xi) f(\xi)-2 g(\xi) f^{\prime}(\xi)\right]
\end{array}\right.
$$

Let $\beta=0$, Then system (8) reduces to

$$
\left\{\begin{array}{l}
f(\tau)+\tau f^{\prime}(\tau)=-\alpha\left[f^{\prime \prime}(\tau)+2 f(\tau) f^{\prime}(\tau)+2 g^{\prime}(\tau)\right], \\
g(\tau)+\frac{1}{2} \tau g^{\prime}(\tau)=\frac{\alpha}{2}\left[g^{\prime \prime}(\tau)-2 g^{\prime}(\tau) f(\tau)-2 g(\tau) f^{\prime}(\tau)\right],
\end{array}\right.
$$


where $\tau=-t^{-\frac{1}{2}} x$, which is a reduction of $\xi$. In facr, system (9) is an ordinary differential system corresponding to the BK system (1).

The group-invariant transformations of the generalized BK system are as follows:

$$
\left\{\begin{array}{l}
g_{1}:(x, t, v, w) \rightarrow(x, t+\epsilon, v, w) \\
g_{2}:(x, t, v, w) \rightarrow(x+\epsilon, t, v, w), \\
g_{3}:(x, t, v, w) \rightarrow\left(2 \beta t e^{\epsilon}+(x-2 \beta t) e^{\frac{1}{2} \epsilon}, t e^{\epsilon}, e^{-\frac{1}{2} \epsilon} v, w e^{-\epsilon}\right) .
\end{array}\right.
$$

In what follows, we consider solutions to the BK system. Set $v=V(\rho), w=W(\rho), \rho=x+l t$. Then (2) becomes

$$
\left\{\begin{array}{l}
l V^{\prime}=\frac{\alpha}{2}\left(V^{\prime \prime}-2 V V^{\prime}-2 W^{\prime}\right)-\beta V^{\prime}, \\
l W^{\prime}=-\frac{\alpha}{2}\left(W^{\prime \prime}+2 W^{\prime} V+2 W V^{\prime}\right)-\beta W^{\prime}
\end{array}\right.
$$

from which we have

$$
\left\{\begin{array}{l}
l V-\frac{\alpha}{2}\left(V^{\prime}-V^{2}-2 W\right)+\beta V=c_{1}, \\
l W+\frac{\alpha}{2}\left(W^{\prime}+2 W V\right)-\beta W^{\prime}=c_{2} .
\end{array}\right.
$$

A special solution to (11) is given by

$$
V=\frac{1}{c+\xi}, \quad W=\frac{1}{(c+\xi)^{2}}
$$

in the case of $l=-\beta, c_{1}=c_{2}=0$. Hence we get a set of solutions to the generalized BK system (2):

$$
v=\frac{1}{c+x-\beta t}, \quad w=\frac{1}{(c+x-\beta t)^{2}} .
$$

Applying the group-invariant transformation (10), we can deduce some other new solutions to system (2):

$$
\begin{cases}g_{1}: & v=\frac{1}{c+x-\beta(t+\epsilon)}, \quad w=\frac{1}{[c+x-\beta(t+\epsilon)]^{2}}, \\ g_{2}: & v=\frac{1}{c+x-\beta t+\epsilon}, \quad w=\frac{1}{(c+x-\beta t+\epsilon)^{2}}, \\ g_{3}: & v=\frac{e^{-\frac{1}{2} \epsilon}}{\left.c+\beta t \epsilon^{\epsilon}+(x-2 \beta t)\right)^{\frac{1}{2} \epsilon}}, \quad w=\frac{e^{-\epsilon}}{\left[c+\beta t e^{\epsilon}+(x-2 \beta t) e^{\frac{1}{2} \epsilon}\right]^{2}} .\end{cases}
$$

Taking $\beta=0$, we can obtain group-invariant solutions to the BK system (1). In particular, we can get the series solutions to the BK system. Indeed, let

$$
f(\tau)=\sum_{n=0}^{\infty} c_{n} \tau^{n}, \quad g(\tau)=\sum_{m=0}^{\infty} c_{m} \tau^{m}
$$


and substituting into (9), we have that

$$
\begin{aligned}
c_{0}+ & \sum_{n=1}^{\infty} c_{n} \tau^{n}+\tau c_{1}+\sum_{n=1}^{\infty}(n+1) c_{n+1} \tau^{n+1} \\
= & -\alpha\left[2 c_{2}+\sum_{n=1}^{\infty}(n+2)(n+1) c_{n+2} \tau^{n}\right]+2\left(c_{0}+\sum_{n=1}^{\infty} c_{n} \tau^{n}\right)\left(c_{1}+\sum_{n=1}^{\infty}(n+1) c_{n+1} \tau^{n}\right) \\
& +2 d_{1}+2 \sum_{m=1}^{\infty}(m+1) d_{m+1} \tau^{m}, \\
d_{0}+ & \sum_{m=1}^{\infty} d_{m} \tau^{m}+\frac{1}{2} \tau d_{1}+\frac{1}{2} \sum_{m=1}^{\infty}(m+1) d_{m+1} \tau^{m+1} \\
= & \frac{\alpha}{2}\left[2 d_{2}+\sum_{m=1}^{\infty}(m+2)(m+1) d_{m+2} \tau^{m}-2\left(c_{0}+\sum_{n=1}^{\infty} c_{n} \tau^{n}\right)\left(d_{1}+\sum_{m=1}^{\infty}(m+1) d_{m+1} \tau^{m}\right)\right. \\
& -2\left(d_{0}+\sum_{m=1}^{\infty} d_{m} \tau^{m}\right)\left(c_{1}+\sum_{n=1}^{\infty}(n+1) c_{n+1} \tau^{n}\right),
\end{aligned}
$$

from which we infer that

$$
\begin{aligned}
c_{2}= & -c_{0} c_{1}-\frac{1}{2 \alpha} c_{0}-d_{1}, \\
d_{2}= & c_{0} d_{1}+d_{0} c_{1}+\frac{1}{\alpha} d_{0} \\
c_{3}= & -\frac{1}{3 \alpha} c_{1}-\frac{2}{3} c_{0} c_{2}-\frac{1}{6} c_{1}^{2}-\frac{2}{3} d_{1}, \\
d_{3}= & \frac{1}{2 \alpha} d_{1}+\frac{2}{3} c_{0} d_{2}-\frac{1}{3} d_{1} c_{1}+\frac{2}{3} d_{0} c_{2}, \\
\ldots . & , \quad\left[-c_{n}-2 \alpha c_{0}(n+1) c_{n+1}-2 \alpha c_{1} c_{n}\right. \\
c_{n+2}= & \frac{1}{\alpha(n+1)(n+2)}\left[\begin{array}{l}
n \\
\quad
\end{array}\right. \\
& \left.\quad+2(n+1) d_{i, j=2}^{n} c_{i} c_{j+1}(j+1) \tau^{i+j}-2 \alpha(n+1) d_{n+1}\right], \\
d_{n+2}= & \frac{1}{(n+1)(n+2)}\left[\frac{2}{\alpha} d_{n}+2(n+1) c_{0} d_{n+1}+2 d_{1} c_{n}+2 \sum_{i, j=2}^{n} c_{i} d_{j+1} \tau^{i+j}\right.
\end{aligned}
$$

where $c_{0}, d_{0}, c_{1}, d_{1}$ are arbitrary parameters. Inserting these expressions into (14), we get the series solutions of the BK system. The second equation of system (9) can be reduced to

$$
g^{\prime \prime}(\tau)-\frac{1}{\alpha} \tau g^{\prime}(\tau)-\frac{2}{\alpha} g(\tau)=0
$$


under the condition

$$
(f g)^{\prime}=0 \quad \Rightarrow \quad f g=c .
$$

As long as the solution of (15) is obtained, we can get the solution $f(\tau)$ from (16). If $g_{1}(\tau)$ is the known solution of (15), then we assume that $g(\tau)=u(\tau) g_{1}(\tau)$. If $u(\tau)$ is known, then the solution $g(\tau)$ to Eq. (15) can be presented. It is easy to see that

$$
g^{\prime \prime}(\tau)=g_{1}(\tau) u^{\prime \prime}(\tau)+2 u^{\prime}(\tau) g_{1}^{\prime}(\tau)+u(\tau) g_{1}^{\prime \prime}(\tau) .
$$

Substituting (17) into Eq. (15) yields

$$
g_{1}(\tau) u^{\prime \prime}(\tau)+\left(2 g_{1}^{\prime}(\tau)-\frac{1}{\alpha} \tau g_{1}(\tau)\right) u^{\prime}(\tau)+\left(g_{1}^{\prime \prime}(\tau)-\frac{1}{\alpha} \tau g_{1}^{\prime}(\tau)-\frac{2}{\alpha} g_{1}(\tau)\right) u(\tau)=0 .
$$

Since

$$
g_{1}^{\prime \prime}(\tau)-\frac{1}{\alpha} \tau g_{1}^{\prime}(\tau)-\frac{2}{\alpha} g_{1}(\tau)=0,
$$

we have

$$
g_{1}(\tau) u^{\prime \prime}(\tau)+\left(2 g_{1}^{\prime}(\tau)-\frac{1}{\alpha} \tau g_{1}(\tau)\right) u^{\prime}(\tau)=0 .
$$

Assume that $u^{\prime}(\tau)=z(\tau)$. Then Eq. (18) becomes

$$
g_{1}(\tau) z^{\prime}(\tau)+\left(2 g_{1}^{\prime}(\tau)-\frac{1}{\alpha} \tau g_{1}(\tau)\right) z(\tau)=0,
$$

which has the solution

$$
z=\frac{c}{g_{1}^{2}(\tau)} e^{\int \frac{1}{\alpha} \tau d \tau}=\frac{c}{g_{1}^{2}(\tau)} e^{\frac{1}{2 \alpha} \tau^{2}}
$$

where $c$ is a constant. Thus we have

$$
\begin{aligned}
& u(\tau)=c \int^{\tau} \frac{1}{g_{1}^{2}(\tau)} e^{\frac{\tau^{2}}{2 \alpha}} d \tau+\bar{c}, \\
& g(\tau)=g_{1}(\tau)\left[c \int^{\tau} \frac{1}{g_{1}^{2}(\tau)} e^{\frac{\tau^{2}}{2 \alpha}} d \tau+\bar{c}\right] .
\end{aligned}
$$

Substituting (19) into Eq. (16), we can get $f(\tau)$. Thus a type of special solutions to system (9) can be obtained.

\section{The self-adjointness of system (2)}

Ibragimov [8] introduced a few related notations of the strict self-adjointness, the nonlinear self-adjointness, and the quasiself-adjointness. Let us recall them.

Let $H$ be a Hilbert space with the scalar product $(u, v)$ defined by

$$
(F u, v)=\left(u, F^{*} v\right), \quad u, v \in H,
$$


where $F^{*}$ is the adjoint operator to a linear operator $F$. A special Hilbert space is given by

$$
H=\left\{\int_{R^{n}}|f(x)|^{2} d x\right\}
$$

along with an inner product

$$
(u, v)=\int_{R^{n}} u(x) v(x) d x .
$$

Let $F$ be a linear differential operator in $H$ whose action on the function $u$ is expressed by $F[u]$. Then Eq. (20) becomes

$$
(F[u], v)=\left(u, F^{*}[v]\right)
$$

which means that

$$
v F[u]-u F^{*}[v]=D_{i}\left(\xi^{i}\right),
$$

where $D_{i}=\frac{\partial}{\partial x^{i}}+u_{i}^{\alpha} \partial_{u^{\alpha}}+u_{i j}^{\alpha} \partial_{u_{j}^{\alpha}}+\cdots$.

For the differential equations

$$
F_{\alpha}\left(x, u, u_{x_{i}}, u_{x_{i} x_{j}}, \ldots\right)=0, \quad \alpha=1, \ldots, m
$$

where $u=\left(u^{1}, \ldots, u^{m}\right)$. The adjoint equations to (22) are as follows:

$$
F_{\alpha}^{*}\left(x, u, v, u_{x_{i}}, v_{x_{i}}, \ldots\right)=0, \quad \alpha=1, \ldots, m
$$

with $F_{\alpha}^{*}=\frac{\delta \varphi}{\delta u^{\alpha}}$. The Lagrangian $\varphi$ for (22) is defined by

$$
\begin{aligned}
& \varphi=\nu^{\beta} F_{\beta}=: \sum_{\beta=1}^{m} \nu^{\beta} F_{\beta}, \\
& \frac{\delta}{\delta u^{\alpha}}=\frac{\partial}{\partial u^{\alpha}}+\sum_{j=1}^{\infty}(-1)^{j} D_{i_{1}} \cdots D_{i_{j}} \frac{\partial}{\partial u_{i_{1} \cdots i_{j}}^{\alpha}} .
\end{aligned}
$$

Definition $1([7,8])$ The differential Eqs. (22) are said to be strictly self-adjoint if their adjoint Eqs. (23) are equivalent to (23) upon the substitution $v=u$. That is, the equation

$$
F^{*}\left(x, u, u, u_{x_{i}}, u_{x_{i}}, \ldots\right)=\lambda F\left(x, u, u_{x}, \ldots\right)
$$

holds with a coefficient $\lambda$.

Definition 2 ([8]) Upon a substitution

$$
v=\varphi(u),
$$

if (23) becomes (22), then we call (22) is quasiself-adjoint. 
Definition $3([7,8])$ Upon a substitution

$$
v=\varphi(x, u) \neq 0
$$

if (26) solves the adjoint Eqs. (23) for all the solutions of (22), then we call system (22) nonlinearly self-adjoint, that is, we have the following equations:

$$
F_{\alpha}^{*}(x, u, \varphi, \ldots)=\lambda_{\alpha}^{\beta} F_{\beta}(x, u, \ldots)
$$

It is easy to find that the strictly self-adjoint and quasiself-adjoint equations both are particular cases of the nonlinear self-adjoint equations.

For the generalized BK system (2), denoted by

$$
\left\{\begin{array}{l}
F=v_{t}-\frac{\alpha}{2}\left(v_{x}-v^{2}-2 w\right)_{x}+\beta v_{x} \\
G=w_{t}+\frac{\alpha}{2}\left(w_{x}+2 w v\right)_{x}+\beta w_{x}
\end{array}\right.
$$

the formal Lagrangian $\mathcal{L}$ can be written as $\mathcal{L}=p F+q G$, and the adjoint system of (2) is as follows:

$$
\left\{\begin{array}{l}
\frac{\delta \mathcal{L}}{\delta v}=2 \alpha p v_{x}-p_{t}-\frac{\alpha}{2} p_{x x}+\alpha(p v)_{x}-\beta p_{x}-\alpha w q_{x}=0 \\
\frac{\delta \mathcal{L}}{\delta w}=-\alpha p_{x}-q_{t}-\alpha(q v)_{x}+\frac{\alpha}{2} q_{x x}-\beta q_{x}=0
\end{array}\right.
$$

Setting $p=\varphi(v, w)$ and $q=\psi(v, w)$ and substituting into (27), along with (28), we have

$$
\left.\frac{\delta \mathcal{L}}{\delta v}\right|_{p=\varphi, q=\psi}=\lambda_{1} F+\mu_{1} G,\left.\quad \frac{\delta \mathcal{L}}{\delta w}\right|_{p=\varphi, q=\psi}=\lambda_{2} F+\mu_{2} G,
$$

where $\lambda_{1}, \lambda_{2}, \mu_{1}, \mu_{2}$ are undetermined functions. It is easy to get

$$
\left\{\begin{array}{l}
p_{t}=\varphi_{v} v_{t}+\varphi_{w} w_{t}, \quad p_{x}=\varphi_{v} v_{x}+\varphi_{w} w_{x} \\
p_{x x}=\varphi_{v v} v_{x}^{2}+2 \varphi_{v w} v_{x} w_{x}+\varphi_{w w} w_{x}^{2}+\varphi_{v} v_{x x}+\varphi_{w} w_{x x} \\
q_{t}=\psi_{v} v_{t}+\psi_{w} w_{t}, \quad q_{x}=\psi_{v} v_{x}+\psi_{w} w_{x} \\
q_{x x}=\psi_{v v} v_{x}^{2}+2 \psi_{v w} v_{x} w_{x}+\psi_{w w} w_{x}^{2}+\psi_{v} v_{x x}+\psi_{w} w_{x x}
\end{array}\right.
$$

Inserting all these results into (29) yields that

$$
\lambda_{1}=\mu_{1}=\lambda_{2}=\mu_{2}=0 .
$$

Therefore, for all solutions of system (2), (28) holds. Thus system (2) is nonlinearly selfadjoint.

\section{Another expression of system (2) and some properties}

Set

$$
v(x, t)=V\left(x, \frac{\alpha}{2} t\right)-\frac{\beta}{\alpha}, \quad w(x, t)=W\left(x, \frac{\alpha}{2} t\right) .
$$


Then system (2) becomes

$$
\left\{\begin{array}{l}
V_{t}=V_{x x}-2 V V_{x}-2 W_{x} \\
W_{t}=-W_{x x}-2 W_{x} V-2 W V_{x}
\end{array}\right.
$$

which has the infinitesimal symmetries

$$
X=\left(2 c_{1} t+c_{2}\right) \partial_{t}+\left(c_{1} x+c_{3} t+c_{4}\right) \partial_{x}+\left(c_{1} v-\frac{1}{2} c_{3}\right) \partial_{V}+2 c_{1} \partial_{W}
$$

where $c_{1}, c_{2}, c_{3}, c_{4}$ are constants. Obviously, when $c_{1}=c_{2}=c_{3}=0$ and $c_{4}=1$, we get $X_{1}=\partial_{x}$. When $c_{1}=c_{3}=c_{4}=0$ and $c_{2}=1$, we have $X_{2}=\partial_{t}$. When $c_{2}=c_{3}=c_{4}=0$ and $c_{1}=1$, we find $X_{3}=2 t \partial_{t}+x \partial_{x}+\partial_{V}+2 \partial_{W} ; X_{i}(i=1,2,3)$ all are particular cases of $X$.

Next, we consider the characteristic equation of $X$ so that we can obtain the similarity reductions of system (30). The characteristic equation of $X$ reads as

$$
\frac{d t}{2 c_{1} t+c_{2}}=\frac{d x}{c_{1} x+c_{3} t+c_{4}}=\frac{d V}{-c_{1} v+\frac{1}{2} c_{3}}=\frac{d W}{-2 c_{1} W} .
$$

Case 1: $c_{1}=1$.

$$
\xi=\frac{x-c_{3} t+c_{4}-c_{2} c_{3}}{\sqrt{2 t+c_{2}}}, \quad V=\frac{1}{2} c_{3}+\frac{f(\xi)}{\sqrt{2 t+c_{2}}}, \quad W=\frac{g(\xi)}{2\left(2 t+c_{2}\right)}
$$

System (30) reduces to

$$
\left\{\begin{array}{l}
-f(\xi)-\xi f^{\prime}(\xi)+2 f(\xi) f^{\prime}(\xi)-f^{\prime \prime}(\xi)+g^{\prime}(\xi)=0 \\
-2 g(\xi)-\xi g^{\prime}(\xi)+g^{\prime \prime}(\xi)+2 g(\xi) f^{\prime}(\xi)+2 g^{\prime}(\xi) f(\xi)=0
\end{array}\right.
$$

Case 2: $c_{1}=c_{2}=0$. Equation (31) becomes

$$
\frac{d t}{0}=\frac{d x}{c_{3} t+c_{4}}=\frac{d V}{\frac{1}{2} c_{3}}=\frac{d W}{0} .
$$

We take

$$
\xi=t, \quad W=W(t), \quad V=\frac{c_{3} x}{2\left(c_{3} t+c_{4}\right)}-\frac{1}{2} c_{3} f(t) .
$$

Then system (30) reduces to

$$
\left\{\begin{array}{l}
c_{3} \xi f^{\prime}(\xi)+c_{3} f(\xi)+c_{4} f^{\prime}(\xi)=0 \\
c_{3} \xi W^{\prime}(\xi)+c_{4} W^{\prime}(\xi)+c_{3} W(\xi)=0
\end{array}\right.
$$

The two equations are in fact the same.

Case 3: $c_{1}=0, c_{2} \neq 0$. Equation (31) reduces to

$$
\frac{d t}{c_{2}}=\frac{d x}{c_{3} t+c_{4}}=\frac{d V}{\frac{1}{2} c_{3}}=\frac{d W}{0} .
$$


We choose

$$
\xi=c_{2} x-\frac{1}{2} c_{3} t^{2}-c_{4} t, \quad V=\frac{c_{3} t}{2 c_{3}}+\frac{f(\xi)}{c_{2}}, \quad W=g(\xi) .
$$

Thus system (30) turns to

$$
\left\{\begin{array}{l}
-2 c_{4} f^{\prime}(\xi)+c_{3}-2 c_{2}^{2} f^{\prime \prime}(\xi)+4 f(\xi) f^{\prime}(\xi)+4 c_{2}^{2} g^{\prime}(\xi)=0 \\
-c_{4} g^{\prime}(\xi)+c_{2}^{2} g^{\prime \prime}(\xi)+2 g^{\prime}(\xi) f(\xi)+2 g(\xi) f^{\prime}(\xi)=0
\end{array}\right.
$$

System (35) has the particular solutions

$$
f(\xi)=\frac{1}{2} \xi^{2}-\xi^{-1}, \quad g(\xi)=c \xi,
$$

where $\xi$ satisfies the constraint

$$
\xi^{3}-\frac{3}{2} \xi^{2}+c-2=0
$$

Thus from (32) we get a set of new solutions of system (2):

$$
\left\{\begin{array}{l}
v(x, t)=\frac{1}{2} c_{3}+\frac{1}{\sqrt{\alpha t+c_{2}}}\left(\frac{1}{2} \frac{\left(x-c_{3} t+c_{4}-c_{2} c_{3}\right)^{2}}{2 t+c_{2}}-\frac{\sqrt{2 t+c_{3}}}{x-c_{3} t+c_{4}-c_{2} c_{3}}\right)-\frac{\beta}{\alpha}, \\
w(x, t)=\frac{c}{2} \frac{x-c_{3} t+c_{4}-c_{2} c_{3}}{\left(\alpha t+c_{2}\right)^{\frac{3}{2}}} .
\end{array}\right.
$$

In what follows, we consider the series solutions of (33).

Setting

$$
f(\xi)=\sum_{i=0}^{\infty} a_{i} \xi^{i}, \quad g(\xi)=\sum_{i=0}^{\infty} b_{i} \xi^{i}
$$

and substituting into system (33), we infer that

$$
\begin{aligned}
& \left\{\begin{array}{l}
a_{0}+2 a_{0} a_{1}-2 a_{2}+b_{1}=0, \\
4 a_{0} a_{2}+2 a_{1}^{2}-6 a_{3}+2 b_{2}=0, \\
-a_{2}+2\left(3 a_{0} a_{3}+3 a_{1} a_{2}\right)-12 a_{4}+3 b_{3}=0,
\end{array}\right. \\
& a_{n}-n a_{n}+2 \sum_{i, j=1}^{n} a_{i}(j+1) a_{j+1}-(n+2) ! a_{n+2}+(n+1) b_{n+1}=0, \\
& \left\{\begin{array}{l}
-2 b_{0}+2 b_{2}+2 b_{0} a_{1}+2 b_{1} a_{0}=0, \\
-3 b_{1}+6 b_{3}+2\left(2 b_{0} a_{2}+b_{1} a_{1}\right)+2\left(b_{1} a_{1}+2 b_{2} a_{0}\right)=0, \\
-4 b_{2}+12 b_{4}+2\left(3 b_{0} a_{3}+2 b_{1} a_{2}+a_{1} b_{2}\right)+2\left(b_{1} a_{2}+2 b_{2} a_{1}+3 b_{3} a_{0}\right)=0, \\
\ldots
\end{array}\right. \\
& -2 b_{n}-(n+1) b_{n+1}+(n+2) ! b_{n+2}+2 \sum_{i, j=1}^{n} b_{i}(j+1) a_{j}+2 \sum_{i, j=1}^{n} a_{i}(j+1) b_{j+1}=0,
\end{aligned}
$$


from which we get

$$
\left\{\begin{array}{l}
a_{2}=\frac{1}{2} a_{0}+a_{0} a_{1}+\frac{1}{2} b_{1} \\
b_{2}=b_{0}-a_{1} b_{0}-a_{0} b_{1} \\
a_{3}=\frac{1}{3}\left(2 a_{0} a_{2}+a_{1}^{2}+b_{2}\right) \\
b_{3}=\frac{1}{2} b_{1}-\frac{1}{3}\left(a_{1} b_{1}+2 a_{2} b_{0}\right)-\frac{1}{3}\left(b_{1} a_{1}+2 b_{2} a_{0}\right) \\
\ldots
\end{array}\right.
$$

where $a_{0}, b_{0}, a_{1}, b_{1}$ are arbitrary parameters. Thus we obtain the following formal series solutions of system (33):

$$
\begin{aligned}
f(\xi)= & a_{0}+a_{1} \xi+\left(\frac{1}{2} a_{0}+a_{0} a_{1}+\frac{1}{2} b_{1}\right) \xi^{2}+\frac{1}{3}\left(2 a_{0} a_{2}+a_{1}^{2}+b_{2}\right) \xi^{3}+\sum_{i=4}^{\infty} a_{i} \xi^{i}, \\
g(\xi)= & b_{0}+b_{1} \xi+\left(b_{0}-a_{1} b_{0}-a_{0} b_{1}\right) \xi^{2} \\
& +\left[\frac{1}{2} b_{1}-\frac{1}{3}\left(2 a_{1} b_{1}+2 a_{2} b_{0}+2 b_{2} a_{0}\right)\right] \xi^{3}+\sum_{i=4}^{\infty} b_{i} \xi^{i},
\end{aligned}
$$

where $a_{i}, b_{i}(i=4,5, \ldots)$ satisfy (36) and (37). Substituting (38)and (39) into (32), we can get the series solutions of the generalized BK system.

Next, we consider the solutions to system (34). It is easy to see that

$$
g(\xi)=f(\xi)=-\xi-\frac{c_{4}}{c_{3}} \quad \text { or } \quad g(\xi)=f(\xi)=\frac{\hat{c}}{\xi+\frac{c_{4}}{c_{3}}},
$$

where $\hat{c}$ is an integration constant.

System (35) is solvable similarly to system (33), and we omit the computations.

\section{Conservation laws}

In this section, we consider the conservation laws of the generalized BK system by using the method in $[7,8]$. From the identity

$$
X+D_{i}\left(\xi^{i}\right)=W^{\alpha} \frac{\delta}{\delta u^{\alpha}}+D_{i} N^{i}
$$

we find that

$$
X(\mathcal{L})+D_{i}\left(\xi^{i}\right) \mathcal{L}=W^{\alpha} \frac{\delta \mathcal{L}}{\delta u^{\alpha}}+D_{i}\left[N^{i}(\mathcal{L})\right]
$$

where

$$
\left\{\begin{array}{l}
X=\xi^{i} \partial_{x_{i}}+\eta^{\alpha} \frac{\partial}{\partial u^{\alpha}}+\xi_{i} \frac{\partial}{\partial u_{i}^{\alpha}}+\cdots, \\
N^{i}=\xi^{i}+W^{\alpha} \frac{\delta}{\delta u_{i}^{\alpha}}+\sum_{s=1}^{\infty} D_{i_{1}} \cdots D_{i_{s}}\left(w_{\alpha}\right) \frac{\delta}{\delta u_{i i_{1} \cdots i_{s}}}, \quad i=1,2, \ldots, n, \\
W^{\alpha}=\eta^{\alpha}-\xi^{j} u_{j}^{\alpha}, \quad \alpha=1, \ldots, m,
\end{array}\right.
$$

and $\mathcal{L}$ is the Euler-Lagrange function, which satisfies

$$
\frac{\delta \mathcal{L}}{\delta u^{\alpha}}=0, \quad \alpha=1, \ldots, m .
$$


Since system (28) holds, we can investigate the conservation laws by using (41), where the components of the conservation laws are the following:

$$
C^{i}=N^{i}(\mathcal{L}), \quad i=1, \ldots, n,
$$

which satisfy the conservation equations

$$
D_{i}\left(C^{i}\right)_{(22)}=0 .
$$

For $X_{1}=\frac{\partial}{\partial x}$, we find that

$$
W^{1,1}=-v_{x}, \quad W^{1,2}=-w_{x} .
$$

Substituting (44) into (42) yields

$$
\left\{\begin{aligned}
C_{v}^{1}= & -\alpha v v_{x}(p+q)-(\alpha+\beta) v_{x} p-\alpha q w v_{x}-\beta q v_{x}+\frac{\alpha}{2} v_{x}\left(q_{x}-p_{x}\right) \\
& +\frac{\alpha}{2} v_{x x}-\frac{\alpha}{2} q v_{x x}, \\
C_{w}^{1}= & -\alpha p v w_{x}-\beta p w_{x}-\alpha q w w_{x}-\alpha p w_{x}-\alpha q v w_{x} \\
& -\beta q w_{x}-\frac{\alpha}{2} p_{x} w_{x}+\frac{\alpha}{2} w_{x} q_{x}+\frac{\alpha}{2} p w_{x x}-\frac{\alpha}{2} q w_{x x} .
\end{aligned}\right.
$$

For $X_{2}=\frac{\partial}{\partial t}$, we get

$$
\left\{\begin{array}{l}
C_{v}^{2}=-v_{t}(p+q)=-(p+q)\left[-\beta v_{x}+\frac{\alpha}{2}\left(v_{x}-v^{2}-2 w\right)_{x}\right] \\
C_{w}^{2}=(p+q)\left[\beta w_{x}+\frac{\alpha}{2}\left(w_{x}+2 w v\right)_{x}\right] .
\end{array}\right.
$$

For $X_{3}=\left(\frac{1}{2} x+\beta t\right) \partial_{x}+t \partial_{t}-\frac{1}{2} v \partial_{v}-w \partial_{w}$, we infer

$$
\left\{\begin{aligned}
& W^{3,1}=-\frac{1}{2} v-t v_{t}-\left(\frac{1}{2} x+\beta t\right) v_{x} \\
& W^{3,2}=-w-t w_{t}-\left(\frac{1}{2} x+\beta t\right) w_{x}, \\
& C_{v}^{3}=\left[-\frac{1}{2} v-t v_{t}-\left(\frac{1}{2} x+\beta t\right) v_{x}\right][\alpha p v+\beta p+2 w q+\alpha p+\alpha q v+\beta \\
& \quad+\frac{\alpha}{2}(p+q)\left[-\frac{1}{2} v_{x}-v_{x t}-\frac{1}{2} v_{x}-\left(\frac{1}{2} x+\beta t\right) v_{x t}\right], \\
& C_{w}^{3}=\left[-w-t w_{t}-\left(\frac{1}{2} x+\beta t\right) w_{x}\right](\alpha p v+\beta p+2 w q+\alpha p+\alpha q v+\beta) \\
&+ \frac{\alpha}{2}(p+q)\left[-w_{x}-t w_{x t}-\frac{1}{2} w_{x}-\left(\frac{1}{2} x+\beta t\right) w_{x x}\right],
\end{aligned}\right.
$$

where $v_{t}, w_{t}$ are given by system (2).

Remark Anco and Bluman [9] proposed a method for constructing conservation laws of differential equations, which uses a formula directly generating the conservation laws and independent of the system having a Lagrangian formulation, in contrast to Noether's theorem, which requires a Lagrangian. They adopted the linear equations and the adjoint equations of the original differential equations to study conservation laws. Essentially, the algorithm presented by Ibragimov et al. is the same as that of Anco and Bluman. Besides, Anco [10] also gave some comments on the work of Ibragimov. 


\section{Conclusions}

In the paper, we have investigated various similarity reductions and exact solutions of the generalized BK system and various its conservation laws by the Lie group analysis. We have pointed out that the standard BK system is only a paticular case of the generalized BK system (2) when $\alpha=-1$ and $\beta=0$. In addition, Lou [11,12] applied the symmetry group method to study some coherent solutions of nonlocal KdV systems and primary branch solutions of a first-order autonomous system. We hope to extend the methods to the systems presented in the paper in the forthcoming days. In addition, Ma [13] obtained some new conservation laws of some discrete evolution equation by symmetries and adjoint symmetries. Zhang, et al. [14, 15] considered symmetry properties of some fractional equations. Therefore there is an open problem how we can look for the fractional systems that correspond to the systems presented in the paper and how we can to solve them. Besides, Liu, Zhang, and Zhou [16] constructed the fractional Volterra hierarchy, gave a definition of the hierarchy in terms of Lax pair and Hamiltonian formalisms, and constructed its tau functions and multisoliton solutions. Bridgman, Hereman, Quispel, and Kamp [17] and El-Nabulsi [18] studied the peakon and Toda lattice. The approaches adopted in [16-18] can lead us to investigate some related properties of the generalized BK system presented in the paper. These questions will be discussed in the future.

\section{Acknowledgements}

The authors wish to thank the anonymous referees for their valuable suggestions.

Funding

This work is supported by the Fundamental Research Funds for the Central University (No. 2017XKZD11).

Availability of data and materials

Not applicable.

Competing interests

The authors declare that they have no competing interests.

Authors' contributions

The authors declare that the study was realized in collaboration with the same responsibility. Both authors read and approved the final manuscript.

\section{Publisher's Note}

Springer Nature remains neutral with regard to jurisdictional claims in published maps and institutional affiliations.

Received: 16 September 2019 Accepted: 18 November 2019 Published online: 04 December 2019

References

1. Broer, L.J.F.: Approximate equations for long water waves. Appl. Sci. Res. 31, 377-395 (1975)

2. Kupershmidt, B.A.: Mathematics of dispersive water waves. Commun. Math. Phys. 99, 51-73 (1985)

3. Kaup, D.J.: A higher-order water-wave equation and the method for solving it. Prog. Theor. Phys. 54, 396-408 (1975)

4. Matveev, V.B., Yavor, M.I.: Solutions presque périodiques et a N-solitons de l'équation hydrodynamique non linéaire de Kaup. Ann. Inst. Henri Poincaré A XXXI (1), 25-41 (1979)

5. Li, Y.S., Ma, W.X., Zhang, J.E.: Darboux transformations of classical Boussinesq system and its new solutions. Phys. Lett. A 275, 60-66 (2000)

6. Olver, P.J.: Applications of Lie Groups to Differential Equations. Springer, New York (1993)

7. Ibragimov, N.H., Avdonina, E.D.: Nonlinear self-adjointness, conservation laws, and the construction of solutions of partial differential equations using conservation laws. Russ. Math. Surv. 68, 889-921 (2013)

8. Ibagimov, N.H.: Nonlinear self-adjointness in constructing conservation laws pp. 1-104 (2011) arXiv:1109.1728vl [math-ph]

9. Anco, S., Bluman, G.: Direct construction of conservation laws from field equations. Phys. Rev. Lett. 78, 2869-2873 (1997)

10. Anco, S.: On the incompleteness of Ibragimov's conservation law theorem and its equivalence to a standard formula using symmetries and adjoint-symmetries. Symmetry 9, 33 (2017)

11. Lou, S.Y., Huang, F.: Alice-Bob physics: coherent solutions of nonlocal KdV systems. Sci. Rep. 7, 1-13 (2016)

12. Lou, S.Y., Yao, R.X.: Invariant functions, symmetries and primary branch solutions of first order autonomous systems. Commun. Theor. Phys. 68, 21-28 (2017) 
13. Ma, W.X.: Conservation laws of discrete evolution equations by symmetries and adjoint symmetries. Symmetry 7 , 714-725 (2015)

14. Zhang, Y.F., et al.: Symmetry properties and explicit solutions of some nonlinear differential and fractional equations. Appl. Math. Comput. 337, 408-418 (2018)

15. Zhang, X.Z., Zhang, Y.F.: Some similarity solutions and numerical solutions to the time-fractional Burgers system. Symmetry 11,112 (2019). https://doi.org/10.3390/sym11010112

16. Liu, S.Q., Zhang, Y., Zhou, C.: Fractional Volterra hierarchy. Lett. Math. Phys. 108, 261-283 (2018)

17. Bridgman, T.J., Hereman, W., Quispel, G.R.W., van der Kamp, P.: Symbolic computation of Lax pairs of partial difference equations using consistency around the cube. Found. Comput. Math. 13, 517-544 (2012)

18. El-Nabulsi, R.A.: Non-standard higher-order G-strand partial differential equations on matrix Lie algebra. J. Niger. Math. Soc. 36, 101-112 (2017)

Submit your manuscript to a SpringerOpen ${ }^{\circ}$ journal and benefit from:

- Convenient online submission

- Rigorous peer review

- Open access: articles freely available online

- High visibility within the field

- Retaining the copyright to your article

Submit your next manuscript at $\gg$ springeropen.com 